\title{
Leptospirosis - A Cause for Concern?
}

\author{
Maj D G Sinclair \\ MRCP(UK), RAMC
}

Senior Registrar

\section{Lt Col M J World}

BSc, MD, MRCP(UK), RAMC

Consultant Physician

Department of Medicine, Cambridge Military Hospital, Aldershot, Hants GU11 $2 \mathrm{AN}$

SUMMARY: A severe case of Weil's disease is presented. The particular relevance of this condition to the Armed Forces and Service physicians is discussed, together with methods of diagnosis.

\section{Introduction}

Leptospirosis remains a rare condition in the United Kingdom, with 50 cases per year on average over the last 10 years (personal communication Dr Ferguson, PHL Hereford); its relevance to military practice is its similarity to established cases of heat injury. This is a report of a confirmed case of Leptospirosis Icterhaemorrhagiae which was initially thought to be severe heat injury. The implications for the soldier and Service physicians are discussed.

\section{Case Report}

A 19 year old recruit who was previously well was admitted to this hospital as an emergency directly from an exercise he had been on for five days in the South Coast area, in early October. On admission he was mildly confused and drowsy but gave a history of vigorous physical exercise undertaken three days before admission, wearing combat kit and webbing and carrying a rucksack and self-loading rifle. Following this he had become unwell, complaining in particular of myalgia in both legs and anuria for the previous 24 hours. He was unable to be accurate about the duration of the preceding physical activity, weather conditions and similar details. No one from the exercise accompanied him to hospital.

There was no history of foreign travel or contact with any infectious diseases. Family history was unremarkable. He was taking no medication and drank alcohol only rarely.

On examination he was markedly jaundiced and clinically dehydrated with a temperature of $38^{\circ} \mathrm{C}$. A small right-sided inferior subconjunctival haemorrhage was noted. There was no lymphadenopathy: cardiovascular and respiratory systems were normal. The abdomen was diffusely tender and the liver just palpable. The spleen was not palpable. Cerebration was sluggish but neurological examination was otherwise normal. The muscles of both legs particularly the quadriceps were swollen and exquisitely tender. Dipstick examination of urine revealed a specific gravity of 1010 with large amounts of blood and protein. An initial diagnosis of heat injury was made although other conditions were not excluded.

Biochemical results on admission and during his stay in hospital are shown in Table 1.

During his eight week admission he suffered from acute tubular necrosis requiring renal support with peritoneal dialysis for 18 days. He also suffered from type one respiratory failure (ie hypoxia with hypocapnia) coincident with a cough productive of haemoptysis and purulent sputum. Transient bilateral infiltrates appeareg on his chest X-ray at this time. Sputum culture gre Streptococcus pneumoniae, sensitive to penicillin which he responded well together with supplementa oxygen and chest physiotherapy. Other problens encountered were of recurring anaemia requiring transfusion due to bleeding into muscles, gut an peritoneum and a severe cholestatic hepatitis and marke rhabdomyolysis.

Only as he recovered did it become apparent that he had not undertaken sufficient strenuous exercise to provoke an illness of this severity due to heat injury alone. Also, he clearly remembered being immersed in stagnant water, some of which he had swallowed on the first occasion whilst on an Assault Course 13 and 16 days prior to feeling unwell. Further investigations were undertaken at this point to determine the cause of the illness.

The diagnosis was made by an enzyme-linked immunosorbent assay for immunoglobulin $\mathbf{M}$ to Leptospira which was positive with a reciprocal titre of 1280 together with a positive leptospira microscopic agglutination test with a reciprocal titre of 5120 for Leptospira icterhaemorrhagiae.

He went on to make a full recovery such that he was able to resume basic training.

\section{Discussion}

This young man suffered from a life threatening episode of classical Weil's disease with myositis, N pneumonitis, peritonitis, nephritis, encephalitis and $\mathrm{N}$ conjunctivitis, the underlying pathology of which is N. thought to be an acute vasculitis causing capillary injury. 
With 50 cases reported per year, leptospirosis remains uncommon in the United Kingdom, although it is thought to be the most widespread zoonosis in the world. The true size of the problem is difficult to assess as many cases are thought to occur as a mild and self-limiting illness never diagnosed as leptospirosis. The disease results from infection from one of the serotypes of the spirochaete Leptospira which are transmitted from animals to humans via contamination of soil and particularly water by infected animal urine. Leptospires do not survive in salt water. The usual portals of entry in humans are abrasions on skin and exposed conjunctival,, 3 nasal and oral mucous membranes, the normal incubation period being 7 to 13 days.

In the past people in occupations associated withes water or sewage were considered to be at particular risk $\overrightarrow{\vec{F}}$ from leptospirosis. Today however the main occupational group at risk are farm workers(1). Water associated activities such as swimming, canoeing and

Table 1

Sequential Biochemical Results During Hospital Admission

Investigation

(Normal Range)

Urea $\mathrm{mmol} /$ litre

$(3-6)$

Creatinine umol/litre

(68-105)

Sodium mmol/litre

(138-145)

Potassium mmol/litre

$(3.3-4.5)$

Creatine Kinase iu/litre

(16-300)

Bilirubin umol/litre

(1.0-17)

Gamma Glutamyl Transferase iu/litre $(3-45)$

Alanine Amino Transferase iu/litre

(4-40)

Prothrombin Time secs

(12-18)

Arterial Partial Pressure Oxygen $\mathrm{kPa}$

$(10.0-13.3)$

Arterial Partial Pressure

Carbon Dioxide $\mathrm{kPa}$

(4.6-6.0)

\begin{tabular}{|c|c|c|c|c|c|c|}
\hline & & & Day of $A$ & Imission & & \\
\hline 1 & 2 & 3 & 6 & 11 & 21 & 6 \\
\hline 5.7 & 30.7 & $50.2(1)$ & 35.6 & 22.3 & $16.6(4)$ & 5.2 \\
\hline 440 & 533 & 726 & 469 & 493 & 254 & 90 \\
\hline 130 & 132 & 123 & 132 & 133 & 141 & 142 \\
\hline 3.8 & 4.7 & 5.6 & 4.9 & 4.4 & 3.9 & $4 \frac{\mathscr{T}}{\overparen{C}}$ \\
\hline 4,800 & 11,141 & 23,000 & 2,721 & 58 & 200 & \\
\hline 190 & 230 & 283 & 526 & 109 & 48 & 14 \\
\hline 28 & 15 & 15 & 24 & 34 & 37 & 7 \\
\hline 173 & 201 & 503 & 289 & 47 & 76 & 40 \\
\hline 15.3 & 16.4 & 17.3 & 13.8 & 13.1 & & \\
\hline 12.6 & 13.6 & $4.8(2)$ & $10.6(3)$ & $10.8(2)$ & & \\
\hline 3.1 & 2.9 & 4.3 & 4.9 & 4.72 & & \\
\hline 7.53 & 7.4 & 7.5 & 7.45 & 7.47 & & \\
\hline
\end{tabular}

Notes:

(1) Peritoneal dialysis started

(2) Breathing air

(4) Peritoneal dialysis stopped

(3) Breathing $40 \%$ oxygen

(5) Discharged from hospital 
similar leisure pursuits are also associated with increased risk.

The similarity of leptospirosis to severe heat injury, a condition seen not uncommonly in military practice, is worth highlighting for two reasons. Firstly there is evidence suggesting that intravenous penicillin, which this patient received fortuitously, is of benefit in severe leptospirosis infection with a shorter duration of illness and curtailment of the duration of renal dysfunction even when given late in the course of the disease(2). Secondly, the future of a recruit in the Army who had suffered an episode of heat injury of this severity is debatable. It is our view that serious consideration should be given to a medical discharge. Indeed, such a course was being considered in this case because of the risk of recurrence until the diagnosis of leptospirosis was made. Service physicians should be aware that soldiers are at risk of leptospirosis by nature of their outdoor training and recreational pursuits and consider the diagnosis in fulminant cases of apparent heat injury, as it can be rapidly confirmed by the Public Health Laboratory at Hereford using an Immunoglobulin Mspecific enzyme-linked immunosorbent assay and a genus specific microscopic agglutination test, $100 \%$ sensitivity being obtainable by performing both tests (3). It may be thought essential that serum samples should be examined for leptospirosis on all patients thought to have heat injury who develop renal failure. The views of colleagues would be welcomed.

It is surprising that there is very little awareness of this potential problem in the Army. Indeed, the Army General and Administrative Instruction (AGAI) on the Hazards of Water does not mention leptospirosis, although it is covered in the section on canoeing. It is our view that the AGAI on the Hazards of Water should be suitably amended, as any activity on or in water, not just canoeing, exposes the participants to the risk of leptospirosis. A suitable warning card is available from the Health and Safety Executive.

\section{Acknowledgements:}

Our grateful thanks to Mrs Pauline Steels for the typing of this manuscript.

\section{REFERENCES}

1. WAITKINS S A. Update on Leptospirosis. Br Med J 1985; 290 : 1502-1503.

2. Watt G, Tuazon M L, Santiago E, et al. Placebocontrolled trial of intravenous penicillin for severe and late Leptospirosis. Lancet 1988; I: 433-435.

3. Watt G, Alquiza L M, Padre L P, Tuazon M L. The rapid diagnosis of Leptospirosis : A prospective compari son of the dot enzyme-linked immunosorbent assay ant5 the genus-specific microscopic agglutination tests at dif ferent stages of illness. $J$ Infect Dis 1988; 4: 840-842 